\title{
Philosophiques
}

\section{Albert Lautman, philosophe des mathématiques}

\section{Jean-Pierre Marquis}

Volume 37, numéro 1, printemps 2010

Albert Lautman, philosophe des mathématiques

URI : https://id.erudit.org/iderudit/039708ar

DOI : https://doi.org/10.7202/039708ar

Aller au sommaire du numéro

Éditeur(s)

Société de philosophie du Québec

ISSN

0316-2923 (imprimé)

1492-1391 (numérique)

Découvrir la revue

Citer ce document

Marquis, J.-P. (2010). Albert Lautman, philosophe des mathématiques.

Philosophiques, 37(1), 3-7. https://doi.org/10.7202/039708ar d'utilisation que vous pouvez consulter en ligne.

https://apropos.erudit.org/fr/usagers/politique-dutilisation/ 


\title{
Albert Lautman, philosophe des mathématiques
}

\author{
JEAN-PIERRE MARQUIS \\ Université de Montréal \\ Jean-Pierre.Marquis@umontreal.ca
}

\begin{abstract}
Albert Lautman, l'homme, le héros de la résistance française, n'a aucunement besoin de présentation. Le philosophe des mathématiques, lui, n'a malheureusement pas reçu l'attention qu'il méritait. Certes, on l'a commenté ponctuellement ici et là, on a heureusement réédité son œuvre récemment, mais jamais n'a-t-on tenté de décrire avec soin la nature du projet philosophique qu'il a tout juste eu le temps d'esquisser durant la dizaine d'années environ avant sa mort tragique en 1944'. Il faut le dire, l'œuvre est rébarbative pour ceux qui ne sont pas prêts à se plonger dans les mathématiques avancées. Car Albert Lautman s'inspirait des développements mathématiques de son époque, suivait le développement des mathématiques en temps réel et inscrivait ces développements dans un cadre philosophique foncièrement original, novateur et, inutile de le cacher, franchement difficile à décoder. On voit en lui un platonicien, mais ce n'est pas le Platon simple et caricatural des Formes ou des Idées extra-mentales et transcendantes. Il explore la dialectique, mais on ne sait trop s'il faut à nouveau remonter à Platon ou si une forme plus contemporaine et originale de dialectique est à l'œuvre. Bref, les obstacles à une compréhension de son approche sont nombreux, difficiles et à la fois mathématiques et philosophiques. Toutefois, sa pensée fascine. Elle ouvre une porte, elle explore des sentiers inconnus, elle articule une synthèse inédite de son maître philosophique, Léon Brunschvicg, qui préconisait une approche résolument historique de la philosophie des mathématiques et de l'école de Hilbert qu'il connaissait bien et finement grâce à son grand ami Jacques Herbrand (et, dans une certaine mesure, de ses contemporains mathématiciens français, dont Élie Cartan, Maurice Fréchet, Claude Chevalley, Charles Ehresmann, les deux derniers comptant également parmi ses bons amis), tout en se positionnant par rapport aux alternatives de l'époque, entre autres celles de ses amis philosophes, cette fois, Jean Cavaillès et Gaston Bachelard, et plus largement, la phénoménologie de Husserl, le premier Heidegger et le Cercle de Vienne, dont il suivait les travaux. Il s'agit là d'un vaste panorama, varié et complexe. Il va sans dire que Lautman prend néanmoins ses distances à la fois de Brunschvicg et de Hilbert et que, si synthèse il y a, elle n'est pas un simple collage, mais plutôt de l'ordre de la synthèse organique, comme la synthèse des protéines, celle qui,
\end{abstract}

1. Emmanuel Barot a, pour la première fois en 2009, publié un livre qui se veut une introduction à l'œuvre philosophique de Lautman. Il en sera question dans la Disputatio que l'on trouvera à la fin de ce numéro. 
à partir d'éléments donnés, génère une entité entièrement nouvelle et originale. La dernière difficulté tient au fait que Lautman a eu bien peu de temps pour développer ses idées et que les textes qu'il a laissés sont bien peu nombreux. J'ai toutefois le plaisir d'annoncer ici une primeur: en effet, grâce aux recherches minutieuses de Fernando Zalamea et à la gentillesse du fils d'Albert, un texte inédit de celui-ci - une œuvre de jeunesse pour ainsi dire - ouvre le présent ouvrage. On y constatera immédiatement l'unité des préoccupations de Lautman au cours de sa trop brève carrière. Ce texte est immédiatement suivi du commentaire de Fernando Zalamea mettant en perspective de manière remarquable et originale les principaux thèmes - en particulier le passage du local au global - qui apparaissent déjà dans ce rapport de 1935, et comment Lautman pressentait, pour ainsi dire, l'idée de faisceau qui allait apparaître sur la scène mathématique à la fin des années quarante et au début des années cinquante ${ }^{2}$.

Les textes présentés ici sont le fruit d'un colloque organisé à l'Université de Montréal au printemps 2008 en l'honneur du 100 anniversaire de naissance d'Albert Lautman. Deux textes se sont ajoutés par la suite, ceux de David Corfield et de Brendan Larvor, deux philosophes des mathématiques anglo-saxons, témoignant ainsi que l'œuvre de Lautman dépasse les frontières de la francophonie. Nous voulions évidemment souligner l'œuvre de Lautman mais également l'interroger, l'interpeller, la mettre en perspective tant historique que conceptuelle, suivre ses traces dans les développements subséquents des mathématiques contemporaines, voir comment marier, si tant faire se peut, une démarche historique et des considérations logiques au sein d'une seule et même approche. Les participants à ce colloque ont tous accepté de relever le défi d'affronter la pensée de Lautman. Voyons brièvement comment chacun a bien voulu le faire.

Les quatre textes qui suivent la discussion du Rapport par Zalamea, ceux de Hourya Benis-Sinaceur, Pierre Cassou-Noguès, Brendan Larvor et David Corfield, abordent tous la question du platonisme de Lautman, une question délicate et difficile s'il en est une, chacun le prenant selon un angle qui lui est propre.

Hourya Benis-Sinaceur aborde de front ce qui constitue probablement le nœud gordien de l'approche lautmanienne, à savoir ce que peut bien être la dialectique selon Lautman et comment elle s'articule avec sa conception des Idées et de leur genèse en mathématiques. Cette exploration fine et subtile de l'œuvre de Lautman nous conduit à travers son platonisme, son utilisation des mathématiques de son époque ainsi que sa lecture d'Heidegger. Benis-Sinaceur propose ici une lecture originale et percutante des travaux du philosophe français qui tente de rendre justice et de mettre à jour la

2. Il faudrait peut-être également explorer dans quelle mesure les développements importants à l'époque en géométrie différentielle, principalement les travaux d'Élie Cartan et de son étudiant Charles Ehresmann, ont pu influencer la réflexion de Lautman sur ce sujet. 
cohérence de la position de Lautman. Cassou-Noguès s'efforce de situer ce platonisme dans la tradition épistémologique française, principalement celle de Brunschvicg, mais également celles de Cavaillès et de Bachelard. Il montre avec une grande acuité les ressemblances et les différences, importantes il faut le souligner, qui séparent Lautman de Brunschvicg et de Cavaillès. Les Idées de Lautman, et par le fait même son platonisme, diffèrent de manière essentielle de leur conception chez son maître et son collègue et ami. Mais cette alternative lautmanienne ne va pas sans difficultés que Cassou-Noguès révèle à juste titre à la fin de sa contribution.

Brendan Larvor examine également le platonisme de Lautman, mais cette fois-ci, non pas en le comparant avec ses contemporains philosophes des mathématiques français, mais plutôt en retournant aux textes de Platon auxquels Lautman fait référence. Larvor tient pour acquis que le dernier texte de Lautman représente la pensée la plus achevée de l'auteur et examine minutieusement les liens faits explicitement par Lautman aux textes de Platon et au texte de Heidegger. Quoique les références à Platon, implicites et explicites, parcourent l'œuvre de Lautman, la référence à Heidegger, elle, est tardive et problématique. Si l'on comprend assez bien à quel Platon Lautman se réfère et de quels interprètes de Platon il s'inspire, on ne sait si l'on doit prendre la référence à Heidegger au sérieux et, si oui, dans quelle mesure elle est essentielle à la compréhension de l'approche lautmanienne. Larvor suggère une lecture de Lautman qui accorde une large part à l'utilisation que celui-ci ferait de Heidegger sur la question du rapport entre la dialectique et les mathématiques.

La contribution de David Corfield vient à son tour enrichir la discussion sur la question de la dialectique et des Idées chez Lautman. Corfield reprend la question du statut de la réalité des Idées, ou la réalité idéale, qui, bien qu'elle s'incarne dans les mathématiques, reste indépendante de ces dernières. Dans un premier temps, Corfield s'interroge sur cette indépendance en soulignant comment certaines idées, qui, à une époque donnée, semblent échapper aux mathématiques, se retrouvent peu après mathématisées complètement. Corfield considère ensuite la possibilité que les mathématiques soient moins homogènes qu'on puisse le croire. On touche ici à la question de l'unité des mathématiques. S'inspirant de commentaires de Timothy Gowers, le médaillé Fields, Corfield suggère que c'est peut-être dans les secteurs de la combinatoire ou de ce que Gowers appelle «les mathématiques des problem-solvers" que l'approche fondamentale de Lautman pourrait être la plus pertinente de nos jours. La question de l'unité des mathématiques nous renvoie directement aux textes de Benis-Sinaceur et de Cassou-Noguès, et il est clair que le dialogue entre ces quatre contributions ne peut que s'enrichir. Si Corfield suggère que certaines idées de Lautman se sont incarnées dans les mathématiques contemporaines, en particulier dans la théorie des catégories, un point de vue qu'il partage avec Fernando Zalamea, Emmanuel Barot, pour sa part, nous convie à mettre côte-à-côte, 
non pas Lautman et Platon, mais plutôt Lautman et Hegel. Ce rapprochement en apparence improbable ouvre cependant des pistes intéressantes et fructueuses. Toutefois, la problématique rejoint celle de Corfield. En effet, si Barot entend mettre en relief les différences et les ressemblances entre les conceptions lautmanienne et hegelienne de la dialectique, son objectif principal est surtout d'examiner les tentatives de formalisation de ces dialectiques et d'en faire ressortir leurs faiblesses. Alors que Corfield maintient que certaines théories mathématiques contemporaines absorbent complètement le point de vue de Lautman, Barot affirme que ces formalisations sont toujours trop courtes. Le débat est lancé.

Yvon Gauthier adopte un point de vue résolument critique à l'égard de Lautman. Il reprend dans une certaine mesure l'idée de Corfield selon laquelle certaines des notions de Lautman se retrouvent aujourd'hui au sein des mathématiques, mais l'enrichit en y ajoutant la logique et le domaine des fondements des mathématiques. Bien que conscient de certains développements de la logique par le biais de son ami Herbrand, Lautman n'aurait toutefois pas pris cette approche suffisamment au sérieux et aurait, par conséquent, été influencé davantage par Brunschvicg que par Herbrand. Gauthier nous présente plusieurs résultats récents des mathématiques et de la logique dont la présentation en termes lautmaniens serait inadéquate. Il évacue également tout rapprochement entre Lautman et Hegel et ne considère pas que le discours philosophique de Lautman permette de mieux comprendre l'objectivité ou l'unité de la connaissance mathématique.

André Lebel nous offre en quelque sorte une synthèse, brève et précise, des problématiques précédentes. Il propose, dans un essai qui s'ouvre sur des interrogations, trois voies à explorer afin de clarifier le rapport entre la dialectique et les mathématiques. Il y a d'abord la voie heideggerienne, et ici il faut certainement se reporter aux textes de Benis-Sinaceur et de Larvor, la voie mathématique, qui nous renvoie cette fois-ci à Corfield et Barot, et, finalement, la voie "problématique » qui est philosophico-mathématique ou mathématico-philosophique puisqu'ici nous avons affaire aux deux côtés d'une seule et même pièce. Dans ce dernier cas, Lebel nous interpelle directement et questionne directement la possibilité, esquissée par Lautman, d'élaborer une théorie des idées. Dans une certaine mesure, le texte de Lebel problématise succinctement et précisément les enjeux soulevés par les textes précédents.

Dans le dernier texte, Mathieu Bélanger reprend la question de l'unité des mathématiques en comparant certaines thèses de Lautman avec celle d'un des mathématiciens les plus importants de la deuxième moitié du $20^{\mathrm{e}}$ siècle, soit Alexandre Grothendieck. Bélanger explore avec soin et précaution les différentes conceptions de l'unité des mathématiques que l'on retrouve chez Lautman. On retrouve ici mis à jour explicitement les ambiguïtés, les flottements et les hésitations typiques de l'œuvre de Lautman. Grothendieck reprend, sans y faire référence, une opposition 
chère à Lautman, celle du discret et du continu. Si l'unité des mathématiques est d'abord et avant tout métaphysique chez Lautman, une affirmation qui reste au demeurant difficile à clarifier pleinement, chez Grothendieck, l'unité du discret et du continu passe par le concept de topos. Bélanger présente comment cette unité est atteinte et quels en sont les avantages. Mais nous faisons de nouveau face aux problèmes soulevés précédemment: les développements mathématiques qui ont suivi les travaux de Lautman effacent-ils pour autant les problématiques qui l'ont intéressé ?

Nous terminons par une disputatio autour du récent ouvrage d'Emmanuel Barot sur Lautman. Après une brève présentation du livre par l'auteur, Pierre Cassou-Noguès, David Corfield et André Lebel offrent quelques pistes de réflexions critiques auxquelles Emmanuel a bien accepté de répondre.

Nous espérons que ce numéro permettra aux lecteurs d'apprécier l'œuvre de Lautman avec un regard neuf et productif. 